\title{
Construção de uma Escala para Avaliação do Planejamento Cognitivo
}

\section{Construction of a Scale to Assess Cognitive Planning}

\author{
Ana Paula Assis de Oliveira \& Elizabeth do Nascimento* \\ Universidade Federal de Minas Gerais, Belo Horizonte, Minas Gerais, Brasil
}

\begin{abstract}
Resumo
Esta pesquisa objetivou construir uma escala de autorrelato para avaliar o planejamento cognitivo em adultos e levantar evidências iniciais de validade. O planejamento foi considerado como um subdomínio das Funções Executivas e entendido como a habilidade de definir e estruturar ações e recursos intencionalmente a fim de atingir um objetivo de maneira eficiente. As etapas envolvidas na construção foram as recomendadas na literatura. Participaram 279 pessoas de ambos os sexos, com diferentes níveis de escolaridade e socioeconômico, residentes em diversos estados brasileiros, com idade entre 18 a 63 anos. A coleta de dados se deu principalmente por meio eletrônico. Os resultados indicam que a Escala de Planejamento Cognitivo (EPC) possui propriedades psicométricas bastante satisfatórias.

Palavras-chave: Planejamento cognitivo, análise psicométrica, avaliação neuropsicológica, funções executivas.

Abstract

This research aimed to construct a self-report scale in order to assess the cognitive planning in adults and to gather initial evidence of its validity. Planning was considered a subdomain of the Executive Functions and it was understood as the ability to intentionally define and structure actions and resources with the purpose of achieving an objective efficiently. The steps of the scale development process were those recommended in the literature. A total of 279 people of both sexes, with different educational and socioeconomic levels, living in several Brazilian states, with ages varying from 18 to 63 years participated in the study. Data collection was performed mainly electronically. Results indicate that the Cognitive Planning Scale (CPS) holds quite satisfactory psychometric properties. Keywords: Cognitive planning, psychometric analysis, neuropsychological evaluation, executive functions.
\end{abstract}

Um construto fundamental no processo de investigação neuropsicológica são as Funções Executivas (FE). Estas podem ser entendidas como um grupo de funções superiores que atuam de forma conjunta e harmoniosa regulando o comportamento humano ao longo do tempo, a fim de atingir objetivos futuros (Barkley, 2010). No geral, as funções integrantes do construto em questão são compostas por processos cognitivos, competências comportamentais e regulação emocional. Na literatura observa-se certa diversificação em relação ao conjunto de subdomínios participantes das FE, contudo boa parte desses componentes se mostra semelhante entre os autores (Anderson, 2008). Segundo Barkley (2010), as FE são compostas pelos seguintes processos: inibição motora, verbal, cognitiva e de atividades emocionais, memória de trabalho não-verbal, memória de trabalho verbal, planejamento, solução de problemas e auto-regulação emocional.

\footnotetext{
* Endereço para correspondência: FAFICH - Av. Presidente Antônio Carlos, 6627, Sala 4048, Campus Pampulha, Belo Horizonte, MG, Brasil, 31270-901. E-mail: anapassis@gmail.com e bethdonascimento@gmail.com
}

Recentemente, as FE têm sido divididas em dois blocos: os componentes frios, que envolvem os processos cognitivos, e os componentes quentes, que são os processos atuantes em situações que demandam a regulação do afeto e da motivação (Anderson, 2008; Chan, Shum, Toulopoulou, \& Chen, 2008). Os componentes frios incluem, entre outros, planejamento, organização, controle inibitório, memória de trabalho e flexibilidade cognitiva. As habilidades integrantes dos componentes quentes são, entre outras, empatia, regulação emocional e tomada de decisão emocional. Cabe ressaltar que essa categorização é apenas teórica, pois essas funções se apresentam interligadas, frequentemente atuando em conjunto na realização das demandas do ambiente (De Luca \& Leventer, 2008).

As FE são imprescindíveis para o gerenciamento efetivo das atividades de vida diária. A fim de interagir de forma funcional com o ambiente é preciso controlar os impulsos, regular a motivação diante de um estímulo fraco ou de uma gratificação distante no tempo, planejar o alcance de objetivos, tomar decisões complexas considerando não só as consequências imediatas, mas também as futuras. Assim 
como é necessário mudar o comportamento ao detectar que este já não é mais adequado, estimar corretamente o tempo na execução das tarefas, manter em mente determinadas informações enquanto realiza simultaneamente alguma outra atividade e regular as emoções nas interações sociais. Todas essas habilidades são fundamentais para o sucesso em qualquer área da vida humana (Malloy-Diniz, Paula, Loschiavo-Alvares, Fuentes, \& Leite, 2010; Malloy-Diniz, Sedo, Fuentes, \& Leite, 2008). O funcionamento saudável das FE nos possibilita atender as demandas do cotidiano de maneira automática sem dimensionarmos a sua importância. De fato, constata-se mais apropriadamente o seu valor quando o seu funcionamento se encontra deficitário (Goldberg, 2002).

Disfunções nessas habilidades acarretam prejuízos significativos impactando o funcionamento pessoal em diferentes contextos. Contudo, o tipo de déficit irá depender de qual subdomínio das FE foi afetado, pois alterações em uma função específica trazem danos singulares no processamento global (Barkley, 2008). Dentre os prejuízos observados podemos citar a incapacidade de iniciar um comportamento, indiferença, dificuldade em mudar o foco da mente, em manter a atenção, comportamento perseverativo, desorganização, falha em aprender com os erros, pobre habilidade de raciocínio, dificuldade em planejar, bem como comportamento social e moral inadequados (Anderson, 2008; Goldberg, 2002). De forma geral, os déficits podem ser representados por cinco dimensões: capacidade de administrar o tempo, de se autoorganizar e solucionar problemas, de se autodisciplinar, de se automotivar e de se autoativar e concentrar (Barkley \& Murphy, 2011). Além de observar disfunções executivas em pacientes com determinados tipos de lesão cerebral, verifica-se a presença de alterações no seu funcionamento em transtornos neurológicos e psiquiátricos como a Doença de Alzheimer, Doença de Parkinson, Esquizofrenia, o Transtorno de Déficit de Atenção/Hiperatividade (TDAH) e o Transtorno Obsessivo-compulsivo (Barkley \& Murphy, 2011; Chan, Chen, Cheung, \& Cheung, 2004; Lange et al., 2003; Rainville et al., 2002; van den Heuvel et al., 2005).

As FE vêm sendo abordadas sob uma perspectiva categorial (funcionamento normal $\mathrm{x}$ funcionamento patológico). Contudo, é importante destacar que a investigação deste construto pode ser conduzida também do ponto de vista de uma habilidade contínua, sem um ponto de corte entre o saudável e o patológico, explorando assim as diferenças individuais no seu desempenho (Pedrero-Pérez et al., 2011). Verdejo-Garcia e Bechara (2010) declaram que as aplicações dos resultados dos estudos das FE vão além da clínica neuropsicológica interessando também à psicopatologia, à psicologia evolutiva e da educação, à psicologia da saúde e ao estudo da adaptação dos mecanismos executivos frente às exigências de um mundo em constante e veloz transformação.

Conforme exposto anteriormente, as FE são um construto composto por vários processos mentais. Todos atuando em conjunto, mas com certo grau de independên- cia (Barkley, 2008). A sua avaliação, por meio de testes, requer o uso de uma bateria formal, construída para tal, ou uma seleção de instrumentos individuais que em seu conjunto, contemplem o exame dessas funções. $\mathrm{O}$ uso de baterias flexíveis permite ao examinador eleger os processos mentais que considera importantes para a sua avaliação (Malloy-Diniz et al., 2010). Em razão de tal fato, os autores do presente trabalho se propuseram a desenvolver um instrumento para avaliar uma função específica das FE, a saber, o planejamento cognitivo.

A proposta de construir uma escala para avaliar o planejamento cognitivo, considerado como um subdomínio das FE tem como meta contribuir para a avaliação refinada e adequada dessa habilidade. $\mathrm{O}$ uso desse recurso se apresenta de grande importância, uma vez que nos permite investigar de maneira detalhada uma variedade de comportamentos, muitos deles dificilmente observados durante a realização de uma tarefa e em um ambiente restrito e com limitação de tempo, como o do consultório. Outro aspecto importante a ser considerado no uso de uma escala é a possibilidade do testando expressar a sua própria percepção sobre a habilidade estudada.

A base teórica da escala de planejamento cognitivo (EPC) foi construída a partir do modelo evolutivo-neuropsicológico das FE do Barkley (1997, 2001, 2006/2008) bem como das contribuições de outros autores em relação à investigação sobre o planejamento cognitivo (Baker et al., 1996; Haddad, 2004; Lezak, 1995; Malloy-Diniz et al., 2010; Owen, 1997; Rowe, Owen, Johnsrude, \& Passingham, 2001; van den Heuvel et al., 2003). Após a análise dos vários estudos selecionados foi possível elaborar uma definição constitutiva e estabelecer os componentes do construto em questão. Portanto, entende-se por planejamento cognitivo a habilidade de definir e estruturar ações e recursos intencionalmente a fim de atingir um objetivo de maneira eficiente. Além da definição, buscou-se por meio desses estudos selecionar os principais aspectos considerados fundamentais para caracterizar o construto em questão. Nessa perspectiva, foram delimitados oito componentes: consciência do objetivo, geração de possibilidades, escolha de uma alternativa, estabelecimento dos passos, antecipação das consequências, hierarquização dos passos, determinação dos recursos e monitoramento das ações.

A principal área cerebral considerada, com significativa concordância, envolvida na habilidade do planejamento é o córtex pré-frontal dorsolateral bilateral. Ela interage com outras áreas corticais e subcorticais formando uma rede que contribui para o planejamento de uma resposta comportamental adequada para se alcançar um objetivo. Contudo, há uma variação entre os estudos em relação a quais seriam essas outras regiões. Dentre as que são apresentadas como candidatas estão: córtex pré-motor bilateral, precuneos bilateral, córtex parietal inferior, área motora suplementar esquerda, córtex insular direito, córtex pré-frontal rostral, striatum direito, córtex cingular anterior rostral e núcleo caudado (Cazalis et al., 2003; Dagher, Owen, Boecker, \& Brooks, 1999; Kaller, Rahm, Spreer, 
Weiller, \& Unterrainer, 2011; Unterrainer et al., 2004; van den Heuvel et al., 2003).

A presença da Neuropsicologia em nosso país é recente e apesar do crescente número de publicações nos últimos anos, ainda se constitui num campo carente de pesquisas. Em que pese o aumento substantivo de demanda por avaliação neuropsicológica, estudos brasileiros de construção, validação e de padronização de testes que possam ser utilizados como recursos auxiliares nesse campo profissional ainda são escassos, sendo de suma importância atuações nesse sentido. Os objetivos desse estudo abarcaram as etapas de construção da Escala de Planejamento Cognitivo e a verificação das propriedades psicométricas do referido instrumento.

\section{Método}

\section{Procedimentos}

A construção da Escala de Planejamento Cognitivo (EPC) foi delineada segundo o modelo de elaboração de instrumental psicológico sugerido por Pasquali (2010). Este modelo é baseado em três pólos: teórico, empírico e analítico. No presente estudo o pólo teórico ficou composto pelas seguintes etapas: definição constitutiva de planejamento e seleção dos componentes por meio de revisão teórica, entrevista com a população meta, construção de itens/instrumento, análise de juízes e análise semântica. O pólo empírico compreendeu a caracterização da amostra e a aplicação da EPC e o pólo analítico abarcou os procedimentos envolvidos na análise psicométrica da EPC.

O projeto foi aprovado pelo Comitê de Ética da Universidade Federal de Minas Gerais (processo ${ }^{\circ}$ 0039.0.203.000-11). Todos os participantes assinaram o Termo de Consentimento Livre e Esclarecido (TCLE).

\section{Procedimentos Teóricos.}

1. Revisão narrativa da literatura. $\mathrm{O}$ método adotado consistiu na busca de artigos da literatura na área de conhecimento 'multidisciplinares' do Portal CAPES, o qual se serviu de várias bases, entre elas a Elsevier, Medline e Springer Link. Foram utilizadas as palavras-chave em inglês: executive functions, planning assessment, cognitive planning, Tower of London e prefrontal cortex. As referências apresentadas nos artigos selecionados serviram de base para busca indireta. Os capítulos de livros de neuropsicologia publicados no Brasil que tratam do tema funções executivas e/ou planejamento também foram revisados. A partir dessas fontes foram selecionados textos em três idiomas: português, inglês e espanhol. Inicialmente a triagem dos artigos foi realizada por meio do resumo. Numa análise mais refinada, os textos selecionados foram aqueles que apresentavam o planejamento como uma das habilidades integrantes das FE ou que o associavam a um sistema neural explorando o seu conceito e as suas características. Foram considerados também estudos que utilizavam o Teste da Torre de Londres, um instrumento amplamente utilizado para avaliar a habilidade em questão. Artigos que tratavam o planejamento cognitivo em outro referencial teórico que não o da Neuropsicologia foram excluídos. Ao final do processo de análise, extraiu-se 25 artigos. No geral, eles abordavam o planejamento numa perspectiva conceitual alinhada, ou seja, continham pontos comuns e complementares. Alguns apresentavam uma definição mais ampla, abarcando vários aspectos, enquanto outros enfatizavam uma ou duas características do planejamento cognitivo. Do total, oito referências foram selecionadas por terem sido consideradas as mais representativas. $\mathrm{O}$ sistema psicológico de interesse foi estabelecido como as FE e o planejamento cognitivo abordado como um subdomínio desse sistema. Nesta etapa foi elaborada uma definição constitutiva de planejamento cognitivo e delimitados os seus componentes, conforme apresentado na introdução.

2. Entrevista com a população meta. Após a revisão teórica elaborou-se um questionário com o objetivo de investigar a definição conceitual de planejamento e quais comportamentos os indivíduos representantes da população meta consideravam relevantes para caracterizar os componentes do planejamento cognitivo. O questionário foi composto por 10 perguntas de resposta livre. Para se chegar à versão final desse questionário, foi realizado um pequeno estudo piloto com cinco participantes. Esse mesmo recurso foi aplicado, de forma individual, em pessoas da comunidade de ambos os sexos, adultas (18 a 59 anos) com diferentes níveis de escolaridade e socioeconômico.

Para análise das respostas dos participantes ao questionário foi utilizado o programa informático Análise Lexical por Contexto de um Conjunto de Segmentos de Texto (ALCESTE) que possibilita a análise quantitativa de dados textuais. De um modo geral, esta ferramenta reconhece o que há de universal frente às várias opiniões relativas a uma temática (Oliveira, Gomes, \& Marques, 2005). Constatou-se que o conteúdo presente nas respostas dos entrevistados se apresentou coerente e homogêneo com a temática proposta, no caso, o planejamento. Os resultados mostraram que a compreensão que representantes da comunidade possuem sobre o conceito de planejamento vem ao encontro com a posição dos especialistas na área. A partir de uma análise qualitativa das respostas dos participantes foi possível verificar que eles identificavam praticamente todos os componentes da habilidade investigada. Tal fato, fez com que o material coletado se tornasse uma fonte rica para a construção de itens constituintes do atual instrumento.

3. Construção de itens/instrumento. Os dados obtidos nas etapas anteriores serviram de fonte para a 
construção dos itens. A proposta inicial foi elaborar um instrumento com número suficiente de itens para avaliação equilibrada de cada componente, contemplando equitativamente tanto a quantidade quanto a direção dos itens. No entanto, dadas as especificidades de cada componente do ponto de vista da definição constitutiva e da manifestação comportamental o que se alcançou não correspondeu a essa proposta inicial. A versão inicial da EPC ficou composta por 89 itens ( 7 itens para Consciência do Objetivo, 11 para Geração de Possibilidades, 9 para Escolha da Ação, 7 para Estabelecimento dos Passos, 12 para Antecipação das Consequências, 12 para Hierarquização dos Passos, 9 para Determinação dos Recursos e 22 para Monitoramento das Ações).

A escala foi organizada em duas partes. A primeira explora comportamentos relacionados com a elaboração do planejamento cognitivo, enquanto a segunda parte os envolvidos na execução do planejamento, sendo esta última composta exclusivamente pelo componente monitoramento das ações. Cada uma das partes inicia com uma expressão geral que intentou diminuir o tamanho dos itens e também evitar a monotonia e o cansaço. Na primeira seção foi exibida a expressão geral 'quando planejo' e na segunda 'quando executo o que planejei'. Essas expressões foram exibidas aproximadamente de 10 em 10 itens a fim de que o testando mantivesse em mente tal afirmação.

As opções de resposta foram elaboradas considerando o critério de frequência, uma vez que os comportamentos sugeridos na escala estão presentes na vida diária. No intuito de proporcionar uma referência para as alternativas propostas, acrescentou-se uma observação ao lado de cada uma, considerando um total de 10 vezes em que a pessoa poderia se encontrar na situação descrita. Assim, cada item deveria ser respondido numa escala likert. As opções de respostas foram: $\mathrm{R}$ - Raramente (em cada 10 situações, comporto dessa forma no máximo 2 vezes), AV- Algumas Vezes (em cada 10 situações, comporto dessa forma de 3 a 4 vezes), MV - Maioria das Vezes (em cada 10 situações, comporto dessa forma de 5 a 7 vezes), QS - Quase Sempre (em cada 10 situações, comporto dessa forma de 8 a 9 vezes) e $\mathrm{S}$ - Sempre (em cada 10 situações, comporto dessa forma as 10 vezes).

4. Análises dos juízes. Com o intuito de verificar se os itens da EPC foram construídos de forma adequada e se representavam o planejamento por meio dos comportamentos sugeridos, três especialistas foram convidados para atuarem como juízes (um psicólogo, doutor em psicologia, com atividade de pesquisa em construção e validade de testes, escalas e outras medidas psicométricas; uma psicóloga, especialista em neuropsicologia e reabilitação neuropsicológica com atuação em avaliação neuropsicológica e um psiquiatra, doutor em neurociências e em psicobiologia com experiência na área de neurociências). Os juízes emitiram o seu parecer em relação aos itens considerando três critérios: identificação do componente representado pelo item, grau de relevância do item e adequação da formulação do item.

A análise quanto à identificação do componente representado pelo item foi conduzida com base no percentual de concordância. Tendo em vista a participação de três especialistas, o percentual adotado foi de no mínimo $67 \%$ de concordância. Verificou-se uma concordância entre os juízes em $90 \%$ dos itens. Entretanto, considerando a concordância entre os juízes sobre a dimensão representada pelo item e a dimensão denominada pelos autores da EPC obteve-se uma concordância de $72 \%$. Cabe ressaltar que dos 25 itens em que não houve concordância entre os especialistas e os autores, 11 estavam relacionados com o monitoramento das ações. A hipótese para o motivo da ocorrência de tal fato é a seguinte: na versão piloto aplicada na população, todos os itens representantes do componente monitoramento das ações compreendem a segunda parte da escala e são precedidos da expressão 'quando executo o que planejei'. Entretanto, no material enviado para os juízes emitirem os seus pareceres na introdução havia uma breve explicação sobre a estruturação da escala esclarecendo que todos os itens da segunda parte seriam precedidos da expressão supracitada, mas não foi determinado quais seriam esses itens. Tal ocorrência dificultou a tarefa de análise para os juízes. Exemplo: no item "Consigo substituir as ferramentas da minha atividade, se for preciso", dois especialistas o identificaram como representando o componente determinação dos recursos e um com o monitoramento das ações. Se o item tivesse sido apresentado de forma completa, "Quando executo o que planejei, consigo substituir as ferramentas da minha atividade, se for preciso"; provavelmente, a sua identificação seria mais precisa.

A análise do grau de relevância do item e adequação da formulação do item foi realizada por meio do Coeficiente de Validade de Conteúdo. Segundo Cassep-Borges, Balbinotti e Teodoro (2010), o ponto de corte recomendado na literatura nesse tipo de análise é 0,80. Entretanto, esses autores ponderam que se os juízes selecionados tiverem formações diferentes pode-se relativizar o ponto de corte. Conforme observado na Tabela 1, 90\% dos itens em relação à relevância e $92 \%$ em relação à adequação obtiveram um CVC acima de 0,70 . Os itens restantes foram analisados qualitativamente. Constatou-se que a divergência de opinião verificada em pelo menos dois dos três juízes ocorreu entre as opções relevante e muito relevante e entre muito adequado e adequado. Sendo assim, os itens foram considerados relevantes e adequados. 
Oliveira, A. P. A. \& Nascimento, E. (2014). Construção de uma Escala para Avaliação do Planejamento Cognitivo.

Tabela 1

Sumário do Coeficiente de Validade de Conteúdo

\begin{tabular}{lcccc}
\hline \multirow{2}{*}{ Faixas CVC } & \multicolumn{2}{c}{ Relevância } & \multicolumn{2}{c}{ Adequação } \\
\cline { 2 - 5 } & $f$ & $\%$ & $f$ & $\%$ \\
\hline$\geq 0,80$ & 63 & 71 & 67 & 75 \\
0,70 a 0,79 & 17 & 19 & 15 & 17 \\
$<0,70$ & 9 & 10 & 7 & 8 \\
Itens CVC $<0,70$ & 80 & 90 & 82 & 92 \\
\hline
\end{tabular}

Além das análises supracitadas, nessa etapa foram levadas em conta algumas considerações apresentadas pelos juízes sobre a formulação e adequação de alguns itens. Como resultado final da etapa de análise de juízes, 11 itens foram modificados e um item eliminado. Assim, a EPC passou a ser composta de 88 itens e não mais de 89 .

5. Análise semântica. Com o objetivo de verificar se os itens foram elaborados de maneira compreensível para a população-meta 20 pessoas foram entrevistadas. O estrato inferior de escolaridade foi composto por oito trabalhadores da construção civil e dois dos serviços gerais. $\mathrm{O}$ estrato superior ficou composto por 10 graduados em quatro diferentes tipos de cursos. A avaliação geral a ser feita com base nas entrevistas foi de que os itens foram compreendidos em sua maioria pelos representantes dos dois estratos. Foram sugeridas alterações na instrução, na formulação de alguns itens e na substituição de algumas palavras. Em síntese, após a análise semântica, a instrução da EPC foi aperfeiçoada por meio da inclusão de uma frase explicativa e 16 itens sofreram alguma modificação, particularmente, substituindo algumas palavras consideradas de difícil entendimento por representantes do estrato inferior.

\section{Procedimentos Empíricos.}

1. Amostra. O tipo de amostra do presente estudo foi de conveniência. Os indivíduos, em sua maioria, representantes da comunidade foram recrutados por meio de uma carta convite enviada por $e$-mail. Uma pequena parcela foi recrutada de uma turma de Educação de Jovens e Adultos de uma escola pública de Belo Horizonte. Participaram do estudo piloto 279 adultos, sendo $54,1 \%$ do sexo feminino. A idade média foi de 32,47 anos, encontrando-se o mais jovem com a idade de 18 anos e o mais velho com a idade de 63 anos. Em relação à escolaridade, a amostra ficou constituída de indivíduos com alta escolaridade, uma vez que a maior parte $(88 \%)$ declarou ter curso superior. A maioria dos participantes declarou residir no estado de Minas Gerais, sendo uma pequena parcela $(9,7 \%)$ moradores de outros 11 estados brasileiros. Sob a perspectiva do nível socio- econômico foram contemplados diferentes níveis. Do total de participantes, $26,2 \%$ reportaram serem portadores de um ou mais transtornos psiquiátricos ou neurológicos, como por exemplo, Ansiedade, Depressão, TDAH, Transtorno Bipolar e Epilepsia. $\mathrm{O}$ uso de medicação por parte desses pacientes foi investigado, porém não foi levado em consideração nessa primeira análise. Nessa fase de construção da escala, o único critério de exclusão foi residir fora do Brasil. Houve a exclusão de um caso.

Tabela 2

Caracterização da Amostra

\begin{tabular}{lccc}
\hline Variável & Categoria & $n$ & $\%$ \\
\hline Sexo & Feminino & 151 & 54,1 \\
& Masculino & 128 & 45,9 \\
Ensino & Fundamental & 06 & 2,2 \\
& Médio & 28 & 10,0 \\
& Superior & 150 & 53,8 \\
& Pós-graduação & 95 & 34,1 \\
Faixa etária & 18 a 29 & 136 & 52,3 \\
& 30 a 63 & 124 & 47,7 \\
\hline
\end{tabular}

2. Coleta de dados da versão piloto da EPC. A coleta de dados foi realizada, em sua maioria, por meio eletrônico. A estrutura do site contemplou a apresentação inicial do TCLE e o consentimento foi obtido via autorização eletrônica. Em seguida, fez-se necessário o preenchimento do questionário de saúde e socioeconômico. Terminada essa etapa inicial, as instruções quanto ao preenchimento da escala foram fornecidas. Os itens da EPC foram aleatorizados, sendo apresentados de forma diferente para cada acesso ao site na tentativa de controlar possíveis vieses de respostas. As páginas foram programadas de modo a buscar garantir que todos os itens fossem respondidos.

Parte dos dados foi obtida através da aplicação manual. As escalas foram preenchidas individualmente, porém em contexto coletivo. Os participantes foram esclarecidos quanto ao objetivo da pesquisa, o sigilo das respostas e o caráter voluntário da participação e em seguida assinaram o TCLE.

\section{Procedimentos Analíticos.}

A análise psicométrica foi conduzida segundo a Teoria Clássica dos Testes (TCT). Inicialmente foram analisados os itens tanto no conjunto geral quanto por componente para avaliação do grau de discriminação. Para tal foi calculada a correlação item-total e analisado o efeito da exclusão do item sobre o índice de consistência interna. Como a escala possui itens formulados na direção inversa, 
antes de proceder às análises, as respostas nesses itens foram invertidas de modo que todas as respostas ficassem na mesma direção. Para investigar a dimensionalidade do instrumento foi utilizada a Análise Fatorial Exploratória (AFE). Para averiguar o nível de fidedignidade da EPC por meio da consistência interna empregou-se o a técnica Alpha de Cronbach.

\section{Resultados}

\section{Análise Psicométrica dos Itens}

As análises dos itens resultaram na eliminação de sete deles (itens 9, 11, 42, 45, 46, 61 e 64) que apresentaram índices de correlação item-total inferiores a 0,30 no conjunto total, mesmo que na análise por componente alguns deles tenham apresentado carga fatorial satisfatória, ou seja, superior a 0,30 . Os itens suprimidos faziam parte dos seguintes componentes: geração de possibilidades (itens 9, 45 e 61), estabelecimento dos passos (item 11), escolha da ação (itens 42 e 46) e antecipação das consequências (item 64). Cabe ressaltar, que mesmo com a exclusão desses itens se garantiu que cada componente ficasse representado por pelo menos cinco itens. O procedimento de exclusão não alterou substantivamente o índice de consistência interna que passou de 0,977 para 0,979, mantendo-se, portanto, bastante alto. Os índices de correlação item-total para o conjunto de 81 itens variou entre 0,32 a 0,77 . Apenas quatro itens obtiveram índices inferiores a 0,40 .

\section{Evidências de Validade por Meio da Estrutura Interna}

Com a finalidade de investigar a estrutura interna da EPC realizou-se uma análise exploratória com os 81 itens por meio do método dos Componentes Principais com rotação Promax. O teste de esferecidade de Bartlett se mostrou significativo ( $\mathrm{x}^{2}$ aproximado de $16554,158, p<$ $0,001)$ e o valor da adequação amostral dos itens, medida pelo teste Kaiser-Meyer-Olkin, foi de 0,953, ambos indicando que a covariância da matriz é bastante acentuada, o que justificou realizar a análise fatorial exploratória. Para definição do número de componentes presentes na matriz foi considerado o autovalor (eigenvalue maior ou igual a 1,0), o percentual da variância explicada, a inspeção visual do gráfico scree plot com base no critério de Cattell (consiste na inspeção visual dos fatores que se encontram à esquerda do ponto em que a curva muda de inclinação "ponto de salto" representando um decréscimo de importância em relação à variância total) e o significado semântico do componente.

Tabela 3

Total da Variância Explicada pelo Método dos Componentes Principais

\begin{tabular}{cccccccc}
\hline & \multicolumn{3}{c}{ Eigenvalues } & & \multicolumn{3}{c}{$\begin{array}{c}\text { Extração } \\
\text { das somas quadradas }\end{array}$} \\
\cline { 2 - 7 } Componente & \multicolumn{3}{c}{$\begin{array}{c}\text { Rotação das } \\
\text { somas quadradas }\end{array}$} \\
\cline { 2 - 7 } & Total & \% da Variância & \% Cumulativa & Total & \% da Variância & \% Cumulativa & Total \\
\hline 1 & 31,13 & 38,44 & 38,44 & 31,13 & 38,44 & 38,44 & 15,55 \\
3 & 6,82 & 8,42 & 46,87 & 6,82 & 8,42 & 46,87 & 23,81 \\
4 & 2,39 & 2,96 & 49,83 & 2,39 & 2,96 & 49,83 & 20,73 \\
5 & 2,05 & 2,53 & 52,36 & 2,05 & 2,53 & 52,36 & 20,21 \\
6 & 1,99 & 2,46 & 54,83 & 1,99 & 2,46 & 54,83 & 20,11 \\
7 & 1,58 & 1,95 & 56,78 & 1,58 & 1,95 & 56,78 & 12,26 \\
8 & 1,43 & 1,77 & 58,55 & 1,43 & 1,77 & 58,55 & 17,40 \\
9 & 1,22 & 1,64 & 60,20 & 1,32 & 1,64 & 60,20 & 13,02 \\
10 & 1,19 & 1,50 & 61,70 & 1,22 & 1,50 & 61,70 & 8,61 \\
11 & 1,14 & 1,41 & 63,18 & 1,19 & 1,47 & 63,18 & 14,05 \\
12 & 1,04 & 1,28 & 64,60 & 1,14 & 1,41 & 64,60 & 4,93 \\
13 & 0,99 & 1,22 & 65,89 & 1,04 & 1,28 & 65,89 & 4,30 \\
\hline
\end{tabular}

Como pode ser observado na Tabela 3, com base no critério do eigenvalue seria possível extrair até 12 componentes da matriz. A inspeção da solução fatorial permitiu visualizar que a maioria dos itens apresentou carga fatorial no primeiro componente e não se identificou uma configu- ração clara sobre o significado semântico de cada um dos 12 componentes. Tal conclusão já era esperada tendo em vista as magnitudes dos eigenvalues e o respectivo percentual da variância explicada que, tal como representado também no gráfico scree plot, apontaram a presença de 
Oliveira, A. P. A. \& Nascimento, E. (2014). Construção de uma Escala para Avaliação do Planejamento Cognitivo.

dois fortes componentes. Tendo em vista que o primeiro componente é aproximadamente cinco vezes maior que o segundo, houve também a indicação forte de que a matriz poderia ser reduzida a uma única dimensão.
Em caráter exploratório foram realizadas análises dos componentes principais para cada um dos componentes do planejamento cognitivo. Todos os componentes se mostraram unidimensionais e sendo representados em geral por itens com cargas fatoriais moderadas a fortes.

Tabela 4

Análises dos Componentes Principais Segundo o Componente do Planejamento Cognitivo

\begin{tabular}{lcccc}
\hline \multicolumn{1}{c}{ Componente } & $\mathrm{N}^{\mathrm{o}}$ de itens & KMO & $\begin{array}{c}1^{\circ} \text { Eigenvalue } \\
\text { (\% variância explicada) }\end{array}$ & $\begin{array}{c}\text { Amplitude das cargas } \\
\text { fatoriais }\end{array}$ \\
\hline Consciência do Objetivo & 7 & 0,89 & $4,19(59,99)$ & 0,57 a 0,88 \\
Geração de Possibilidades & 7 & 0,87 & $3,79(54,25)$ & 0,37 a 0,62 \\
Escolha da Ação & 8 & 0,84 & $3,39(42,45)$ & 0,39 a 0,79 \\
Estabelecimento dos Passos & 5 & 0,79 & $2,70(54,08)$ & 0,51 a 0,83 \\
Antecipação das Consequências & 10 & 0,88 & $4,49(44,92)$ & 0,44 a 0,77 \\
Hierarquização das Passos & 13 & 0,93 & $6,68(51,43)$ & 0,58 a 0,83 \\
Determinação dos Recursos & 9 & 0,87 & $3,81(42,35)$ & 0,38 a 0,80 \\
Monitoramento das Ações & 22 & 0,93 & $9,44(42,93)$ & 0,40 a 0,76 \\
\hline
\end{tabular}

Tabela 5

Exemplos de Itens e Respectivas Cargas Fatoriais

Item

Carga Fatorial

Tenho clareza do meu objetivo.

0,66

Identifico previamente os passos que devem ser seguidos a fim de atingir o objetivo.

0,78

Mantenho em mente aonde quero chegar.

0,71

Faço escolhas erradas.

0,42

Procuro estabelecer a sequência mais adequada das ações.

0,75

Penso em cima da hora nos recursos que preciso para realizar uma tarefa. $\quad 0,55$

Procuro refletir sobre possíveis maneiras de resolver um problema.

0,68

Consigo pensar nos vários aspectos envolvidos com intuito de escolher a melhor saída. $\quad 0,70$

Acabo envolvendo-me com outras atividades perdendo de vista o objetivo principal. $\quad 0,54$

$\begin{array}{ll}\text { Procuro ter uma noção geral da tarefa ao pensar nas várias possibilidades. } & 0,67\end{array}$

$\begin{array}{ll}\text { Verifico se uma ação depende da outra. } & 0,73\end{array}$

$\begin{array}{ll}\text { Sistematizo as ações que devo realizar. } & 0,71\end{array}$

Verifico se disponho dos recursos necessários para realizar uma tarefa. $\quad 0,72$

$\begin{array}{ll}\text { Eu traço previamente estratégias para realizar uma tarefa complexa. } & 0,76\end{array}$

Utilizo a primeira solução que me vem à mente mesmo sendo para uma tarefa difícil. $\quad 0,45$

Tenho dificuldade em adotar algum critério para organizar as minhas ações. $\quad 0,57$

$\begin{array}{ll}\text { Estabeleço prioridades das etapas. } & 0,72\end{array}$

Lido bem com os imprevistos. $\quad 0,38$

Tenho dificuldade em manter o curso das minhas ações. $\quad 0,60$

Fico preso a uma parte do plano, o que acaba prejudicando o resultado final. $\quad 0,43$

Nota. Cargas fatoriais da solução unifatorial pelo método PAF. 
Três análises exploratórias pelo método Principal Axis Factoring (PAF) foram conduzidas: com oito fatores, tal como proposto no processo de construção da escala, com dois fatores conforme indicação dos resultados da primeira análise e finalmente com apenas um fator. Como resultado, a análise de oito fatores não se mostrou pertinente. A solução de dois fatores revelou que o segundo fator ficou composto exclusivamente dos itens elaborados na direção oposta aos demais apresentando cargas fatoriais importantes também no primeiro fator. Por fim, a análise fatorial exploratória final pertinente foi a unifatorial.

Em geral, as cargas fatoriais dos itens se mostraram adequadas indicando que eles se constituem em ótimos representantes comportamentais do planejamento cognitivo. A maioria dos itens (51\%) exibiu cargas fatoriais variando entre 0,60 a 0,79 e apenas cinco itens apresentaram cargas fatoriais entre 0,30 a 0,39 . Na Tabela 5 podem ser verificados alguns itens da EPC com as suas respectivas cargas fatoriais.

\section{Discussão e Conclusão}

Os testes são ferramentas de extrema importância na investigação neuropsicológica, uma vez que nos fornecem dados objetivos para a compreensão clínica. No caso do planejamento cognitivo, os instrumentos utilizados para a sua avaliação podem fazer parte de uma bateria formal seja para o exame das Funções Executivas como o Teste do Mapa do Zoológico da Behavioral Assessment of the Dysecutive Syndrome ou do exame da inteligência como o subteste Labirintos da Escala Wechsler de Inteligência para Crianças (WISC-III). E também, através de testes isolados como: Teste da Torre de Londres (TOL), Teste da Torre de Hannoi, Porteus Maze Test (Malloy-Diniz et al., 2010; Lezak, 1995). A Figura Complexa de Rey não foi criada originalmente para se avaliar o planejamento cognitivo. No entanto, há proposições de modelos como o de Waber e Holmes, o Modelo Boston Qualitative Scoring System e o de Savage e colaboradores para se investigar essa função por meio desse teste (Fuentes, D'Alcante, \& Savage, 2010). O TOL é um teste neuropsicológico bem consolidado para avaliação do planejamento cognitivo. No Brasil, existem estudos publicados considerando o desempenho no TOL em diferentes níveis de desenvolvimento: em crianças (Malloy-Diniz et al., 2008), em adultos (Souza, Ignácio, Cunha, Oliveira, \& Moll, 2001) e em idosos (Moreira, Malloy-Diniz, Fuentes, Correa, \& Lage, 2010). No entanto, as limitações observadas em relação a este teste é que ele possui um teto insuficiente e não apresenta validade ecológica, ou seja, os estímulos propostos são distantes da realidade vivenciada pelo testando. Desenvolver instrumentos de avaliação que apresentem validade ecológica tem sido uma crescente preocupação no campo da neuropsicologia.

Dentre os testes psicológicos, escalas tanto de auto quanto de heterorrelato se apresentam como outro recurso utilizado para investigar as FE e por extensão o planeja- mento como um subdomínio. Comumente, ela é estruturada para avaliar de forma geral as habilidades selecionadas pelo autor como integrantes das FE. Como por exemplo, o Questionário de Disfunções Executivas (Pedrero-Pérez et al., 2011) e a Escala de Déficits no Funcionamento Executivo (Barkley \& Murphy, 2011). Nestes instrumentos são verificadas poucas questões explorando aspectos relacionados com o planejamento cognitivo.

Com o intuito de disponibilizar um instrumento construído a partir da realidade brasileira foi desenvolvida a Escala de Planejamento Cognitivo (EPC). Neste recurso buscaram-se explorar de uma maneira minuciosa os oitos aspectos considerados importantes para a caracterização da habilidade em foco. Os itens foram construídos baseando-se principalmente nas respostas coletadas com a população meta, as quais se mostraram próximas da visão dos especialistas. A estratégia de se usar um recurso textual para verificar como o planejamento é percebido e relatado, além de ter proporcionado uma abundância de itens, também cumpriu o objetivo de garantir uma maior validade ecológica da escala.

A análise psicométrica da EPC se mostrou bastante satisfatória. A sua composição inicial foi de 88 itens a serem respondidos numa escala tipo likert, considerando o critério de frequência. Pasquali (2010) declara que se a elaboração do instrumento foi bem trabalhada nas suas fases iniciais, bem fundamentada teoricamente, a versão piloto não precisa iniciar com mais de $10 \%$ dos números de itens que se deseja salvar. Com base na análise dos itens (correlação item-total) foram eliminados 7 itens, o que corresponde a $8 \%$ do total. Em relação à consistência interna identificou-se alto grau de precisão. Em termos da estrutura interna, a análise fatorial indicou a EPC como unidimensional com cargas fatoriais adequadas. Tal solução mostra que num processo de normatização será justificada somente a geração de um escore global. No entanto, a Análise Fatorial Exploratória por componente revelou que cada um deles se encontra adequadamente representado do ponto de vista psicométrico. Sendo assim, será viável ao profissional realizar uma análise qualitativa das respostas, a partir dos componentes, identificando possíveis forças e fraquezas no planejamento que possam servir de fonte de informações mais específicas para elaboração de um processo diagnóstico e de intervenção.

Estudiosos das FEs declaram que os dois métodos utilizados para avaliá-las, escalas e testes, contribuem de formas distintas na predição de prejuízos no funcionamento ocupacional. As escalas se mostram significativamente superiores em tal aspecto (Barkley \& Fischer, 2011; Barkley \& Murphy, 2010). Para esses autores, tal discrepância acontece, possivelmente, pelo fato de cada uma das técnicas avaliarem diferentes níveis de um sistema de FE hierarquicamente organizado. Os testes avaliariam o nível mais básico e as escalas níveis mais elevados dessa hierarquia, que abrangem sequências comportamentais voltadas para o alcance de objetivos mais complexos, que demandam uma rede social mais ampla e que precisam ser 
sustentadas por um período de tempo mais prolongado. Acredita-se que a EPC consiga abarcar as competências necessárias para o alcance de objetivos mais complexos. Sendo assim, é possível que ela se apresente como um bom instrumento para predizer o desempenho nas demandas ocupacionais. Futuras investigações serão necessárias para comprovar esta hipótese.

Esse estudo apresenta algumas limitações. Uma delas envolve a composição da amostra em relação ao nível de escolaridade. Aproximadamente $88 \%$ dos participantes declararam possuir nível superior. Sendo que desse total, cerca de um terço é pós-graduado. Portanto, há a necessidade de se ampliar a coleta de dados em outros níveis de escolaridade como ensino fundamental e médio. Outra limitação se relaciona com a técnica estatística utilizada para análise dos itens, no caso, a TCT. Atualmente, pode-se contar com os avanços no campo da psicometria para análise dos itens como as técnicas oferecidas pela Teoria de Resposta ao Item. Num futuro breve, pretende-se realizar um estudo comparativo sobre a análise dos dados utilizando tanto da TCT quanto da TRI.

Tendo em mente as limitações, os resultados favoráveis verificados na versão piloto da EPC constituem em estímulo para avançar no processo de desenvolvimento dessa escala. É possível que ela seja reduzida de forma a tentar uma proporcionalidade na quantidade de itens que representam cada componente. Isso poderá ser feito com base em alguns critérios: índices psicométricos, formulação da frase e decisão qualitativa. Pretende-se realizar novos estudos de levantamento de validade e ao alcançar a versão final proceder o estabelecimento de normas com uma amostra representativa da população. Acredita-se que a conclusão do processo de desenvolvimento da EPC possa colaborar para, entre outros, diagnósticos mais refinados, elaboração de programas de reabilitação mais específicos e identificação de diferenças individuais no seu desempenho. Dessa forma, poderá contribuir para a melhora de tomadas de decisões na prática profissional.

\section{Referências}

Anderson, P. J. (2008). Towards a developmental model of executive functions. In V. Anderson, R. Jacobs, \& P. J. Anderson (Eds.), Executive functions and the frontal lobes: A lifespan perspective (pp. 23-56). New York: Taylor \& Francis.

Baker, S. C., Rogers, R. D., Owen, A. M., Frith, C. D., Dolan, R.J., Frackowiak, R. S. J., \& Robbins, T. W. (1996). Neural systems engaged by planning: A PET study of the Tower of London task. Neuropsychologia, 34(6), 515-526.

Barkley, R. A. (1997). Behavioral inhibition, sustained attention, and executive functions: Constructing a unifying theory of ADHD. Psychological Bulletin, 121(1), 65-94.

Barkley, R. A. (2001). The executive functions and self-regulation: An evolutionary neuropsychological perspective. Neuropsychology Review, 11(1), 1-29.

Barkley, R. A. (2008). Uma teoria para o TDAH. In R. Barkley, Transtorno de déficit de atenção/hiperatividade: Manual para diagnóstico e tratamento (3. ed., R. Costa, trad., Cap. 7, pp. 309-348). Porto Alegre, RS: Artmed. (Original publicado em 2006)
Barkley, R. A. (2010). Differential diagnosis of adults with ADHD: The role of executive function and self-regulation [Abstract]. Journal of Clinical Psychiatry, 71.

Barkley, R. A., \& Fischer, M. (2011). Predicting impairment in major life activities and occupational functioning in hyperactive children as adults: Self-reported executive function (EF) deficits versus EF tests. Developmental Neuropsychology, 36(2), 137-161.

Barkley, R. A., \& Murphy, K. R. (2011). The nature of executive function (EF) deficits in daily life activities in adults with ADHD and their relationship to performance on EF Tests. Journal of Psychopathology and Behavioral Assessment, 33(2), 137-158

Cassep-Borges, V., Balbinotti, M. A. A., \& Teodoro, M. L. M. (2010). Tradução e validação de conteúdo: Uma proposta para a adaptação de instrumentos. In L. Pasquali (Ed.), Instrumentação psicológica: Fundamentos e práticas (pp. 506-520). Porto Alegre, RS: Artmed.

Cazalis, F., Valabrègue, R., Pélégrini-Issac, M., Asloun, S., Robbins, T. W., \& Granon, S. (2003). Individual differences in prefrontal cortical activation on the Tower of London planning task: Implication for effortful processing. European Journal of Neuroscience, 17, 2219-2225.

Chan, R. C. K., Chen, E. Y. H., Cheung, E. F. C., \& Cheung, H. K. (2004). Executive dysfunctions in schizophrenia: Relationships to clinical manifestation. European Archives Psychiatry and Clinical Neuroscience, 254(4), 256-262.

Chan, R. C. K., Shum, D., Toulopoulou, T., \& Chen, E. Y. H. (2008). Assessment of executive functions: Review of instruments and identification of critical issues. Archives of Clinical Neuropsychology, 23, 201-216.

Dagher, A., Owen, A. M., Boecker, H., \& Brooks, D. J. (1999). Mapping the network for planning: A correlational PET activation study with the Tower of London task. Brain, 122, 1973-1987.

De Luca, C. R., \& Leventer, R. J. (2008). Developmental trajectories of executive functions across the lifespan. In V. Anderson, R. Jacobs, \& P. J. Anderson (Eds.), Executive functions and the frontal lobes: A lifespan perspective (pp. 23-56). New York: Taylor \& Francis.

Fuentes, D., D’Alcante, C. C., \& Savage, C. (2010). Planejamento cognitivo da ação através da Figura Complexa de Rey. In L. F. Malloy-Diniz, D. Fuentes, P. Mattos, \& N. Abreu (Eds.), Avaliação neuropsicológica (pp. 355-361). Porto Alegre, RS: Artmed.

Goldberg, E. (2002). O cérebro executivo: Lobos frontais e a mente civilizada (R. Fiker \& M. E. Fiker, trads.). Rio de Janeiro, RJ: Imago. (Original publicado em 2001)

Haddad, F. A. (2004). Planning versus speed: An experimental examination of what Planned Codes of the Cognitive Assessment System measures. Archives of Clinical Neuropsychology, 19, 313-317.

Kaller, C. P., Rahm, B., Spreer, J., Weiller, C., \& Unterrainer, J. M. (2011). Dissociable contributions of left and right dorsolateral prefrontal cortex in planning. Cerebral Córtex, 21, 307-317.

Lange, K. W., Tucha O., Alders, G. L., Preier, M., Csoti, I., Merz, B., ...Naumann, M. (2003). Differentiation of parkinsonian syndromes according to differences in executive functions. Journal of Neural Transmission, 110, 983-995.

Lezak, M. D. (1995). Executive functions and motor performance. In Neuropsychological assessment ( $3^{\text {rd }}$ ed., pp. 650-685). Oxford, UK: Oxford University Press. 
Malloy-Diniz, L. F., Paula, J. J., Loschiavo-Alvares, F., Fuentes, D., \& Leite, W. B. (2010). Exame das funções executivas . In L. F. Malloy-Diniz, D. Fuentes, P. Mattos, \& N. Abreu (Eds.), Avaliação neuropsicológica (pp. 94-113). Porto Alegre, RS: Artmed.

Malloy-Diniz, L. F., Sedo, M., Fuentes, D., \& Leite, W. B. (2008). Neuropsicologia das funções executivas. In D. Fuentes, L. F. Malloy-Diniz, C. H. P. Camargo, \& R. M. Cosenza, Neuropsicologia: Teoria e prática (pp. 187-206). Porto Alegre, RS: Artmed.

Moreira, A. G. G., Malloy-Diniz, L. F., Fuentes, D., Correa, H., \& Lage, G. M. (2010). Atividade física e desempenho em tarefas de funções executivas em idosos saudáveis: Dados preliminares. Revista de Psiquiatria Clínica, 37(3), 109-112.

Oliveira, D. C., Gomes, A. M. T., \& Marques, S. C. (2005). Análise estatística de dados textuais na pesquisa das representações sociais: Alguns princípios e uma aplicação ao campo da saúde. In M. S. S. Menin \& A. M. Shimizu (Eds.), Experiência e representação social: Questões teóricas e metodológicas (pp. 157-200) São Paulo, SP: Casa do Psicólogo.

Owen, A. M. (1997). Cognitive planning in humans: Neuropsychological, neuroanatomical and neuropharmacolgical perspectives. Progress in Neurobiology, 53, 431-450.

Pasquali, L. (2010). Testes referentes a construto: Teoria e modelo de construção. In L. Pasquali (Ed.), Instrumentação psicológica: Fundamentos e práticas (pp. 165-198). Porto Alegre, RS: Artmed.

Pedrero-Pérez, E. J., León, J. M. R.-S., Lozoya-Delgado, P., Llanero-Luque, M., Rojo-Mota, G., \& Puerta-García, C. (2011). Evaluación de lós sintomas prefrontales: propiedades psicométricas y datos normativos del cuestionario disejecutivo (DEX) en una muestra de población espanõla. Revista de Neurología, 52(7), 394-404.

Rainville, C., Amieva, H., Lafont, S., Dartigues, J. F., Orgogozo, J. M., \& Fabrigoule, C. (2002). Executive function deficits in patients with dementia of the Alzheimer's type: A study with a Tower of London task. Archives of Clinical Neuropsychology, 17, 513-530.

Rowe, J. B., Owen, A. M., Johnsrude, I. S., \& Passingham, R. E. (2001). Imaging the mental components of a planning task. Neuropsychologia, 39, 315-327.

Souza, R. O., Ignácio, F. A., Cunha, F. C. R., Oliveira D. L. G., \& Moll, J. (2001). Contribuição à neuropsicologia do comportamento executivo: Torre de Londres e Teste de Wisconsin em indivíduos normais. Arquivos de Neuropsiquiatria, 59(3-A), 526-531.

Unterrainer, J. M., Rahm, B., Kaller, C. P., Ruff, C. C., Spreer, J., Krause, B. J., ...Halsband, U. (2004). When planning fails: Individual differences and error-related brain activity in problem solving. Cerebral Cortex, 14, 1390-1397.

van den Heuvel, O. A., Groenewegen, H. J., Barkhof, F., Lazeron, R. H. C., Dyck, R., \& Veltman, D. J. (2003). Frontostrialtal system in planning complexity: A parametric functional magnetic resonance version of Tower of London task. NeuroImage, 18, 367-374.

van den Heuvel, O. A., Veltman, D. J., Groenewegen, H. J., Cath, D. C., Balkom, A. J. L. M., Hartskamp, J., ...Dyck, R. (2005). Frontal-striatal dysfunction during planning in obsessive-compulsive disorder. Archives of General Psychiatry, 62, 301-310.

Verdejo-Garcia, A., \& Bechara, A. (2010). Neuropsicología de las funciones ejecutivas. Psicothema, 22, 227-235. 